\title{
Influence of flattening contralateral head-related transfer functions upon sound localization performance
}

\author{
Kanji Watanabe ${ }^{1, *}$, Ryosuke Kodama $^{2}$, Sojun Sato ${ }^{1, \dagger}$, Shouichi Takane ${ }^{1, \ddagger}$ and Koji Abe ${ }^{1, \S}$ \\ ${ }^{1}$ Faculty of Systems Science and Technology, Akita Prefectural University \\ ${ }^{2}$ Graduate School of Systems Science and Technology, Akita Prefectural University
}

(Received 19 October 2010, Accepted for publication 27 December 2010)

Keywords: HRTF, Localization, Spectral cue, Contralateral side PACS number: 43.60.-c, 43.66. $-\mathrm{x} \quad$ [doi:10.1250/ast.32.121]

\section{Introduction}

The human auditory system can localize sound sources by using various physical cues, such as interaural time difference (ITD), interaural level difference (ILD), and spectrum. The ITD and ILD are known to be reliable cues for sound localization in azimuth. For localizing elevation or discriminating front from back, spectral cues are very important [1]. Investigations to clarify the role of spectral cues have been conducted by many researchers. One approach is to use bandlimited stimuli. Blauert [2] found that localization judgments in the median plane were related to the center frequency of the stimuli. Hebrank and Wright [3] found that frontal elevation was cued by the lower cutoff frequency of a 1-octave notch whose center frequency was varied from $4 \mathrm{kHz}$ to $10 \mathrm{kHz}$, and that back elevation was cued by a small peak from $10 \mathrm{kHz}$ to $12 \mathrm{kHz}$. Another approach is to use broadband stimuli. In particular, many investigations have used head-related transfer functions (HRTFs), which are known to comprehensively include localization cues [1]. Wightman and Kistler [4] reported that front-back confusion was related to highfrequency cues derived from the anterior-posterior asymmetry of the pinna. Some studies have tried to clarify spectral cues by deteriorating HRTFs. Asano et al. [5] reported that smoothing of the frequency characteristics of HRTFs in highfrequency regions did not affect localization performance in the median plane. Kistler and Wightman [6] used HRTFs modeled by low-order ( $1 \mathrm{st}-5$ th) principal components to show that the primary peaks and dips in high-frequency regions were important for front-back discrimination. Iida et al. [7] represented HRTFs with one peak and two notches and demonstrated via psycho-acoustical experiment that the simplified HRTFs controlled median plane localization well. Langendijk and Bronkhorst [8] removed cues from certain HRTF frequency bands by flattening magnitude characteristics of the bands. They reported that front-back confusion was significantly increased when spectral cues in highfrequency regions were removed.

In most of these investigations, localization cues were equally removed from or deteriorated for both right-ear and left-ear HRTFs. However, the frequency characteristics of

\footnotetext{
*e-mail: kwatanabe@akita-pu.ac.jp

†e-mail: sojun_sato@akita-pu.ac.jp

†e-mail: takane@akita-pu.ac.jp

§e-mail: koji@akita-pu.ac.jp
}

HRTFs and temporal waveforms of head-related impulse responses (HRIRs) generally show remarkable differences between ears. Therefore, removing cues from the HRTF of either ear might show different influences from those in previous studies. It can generally be said that in comparison with the HRTFs on the ipsilateral side, those on the contralateral side have a lower energy and a more complicated spectral form with many peaks and dips. It is also obvious that the frequency resolution of the human auditory system is relatively rough in the high-frequency region [9]. Taking these two points together, the detailed spectral form of the contralateral HRTFs in high frequency regions is unlikely to be perceived. It remains unclear, however, whether those peaks and dips are required as localization cues if the HRTFs on the ipsilateral side and the binaural cues are accurately reproduced.

To further explore this issue, we investigated the influence of flattening contralateral HRTFs. As described herein, a subjective evaluation was conducted using HRTFs on the contralateral side, whose spectral form was flattened in regions above a given frequency.

\section{Method of flattening contralateral HRTFs}

Our method of removing spectral cues from contralateral HRTFs was similar to that used by Langendijk and Bronkhorst [8], except that they used the method for both ears. Magnitude characteristics of the measured HRTFs on the contralateral side were flattened above a certain frequency. This frequency is referred to as the "boundary frequency" in this paper. The amplitude level of the flattened region was set to the average level of the original HRTF in the corresponding frequency region. This method preserved the ILD calculated in the overall frequency range. The initial time delay included in each processed HRIR was adjusted so that it had the same value as that included in the corresponding HRIR before flattening. Thus, the ITD was also maintained. This processing does not accurately retain the frequency characteristics of the ILD and ITD but does preserve the binaural cues as much as possible. An example of the method is presented in Fig. 1. Figure 1(a) shows the frequency characteristics of a specific original HRTF. Figure 1(b) shows that the spectral form was flattened in the region of frequencies higher than the boundary frequency of $8 \mathrm{kHz}$. The relative level in this region, about $-2.8 \mathrm{~dB}$ was equal to the average level of the original HRTF in the frequency region corresponding to the flattened 


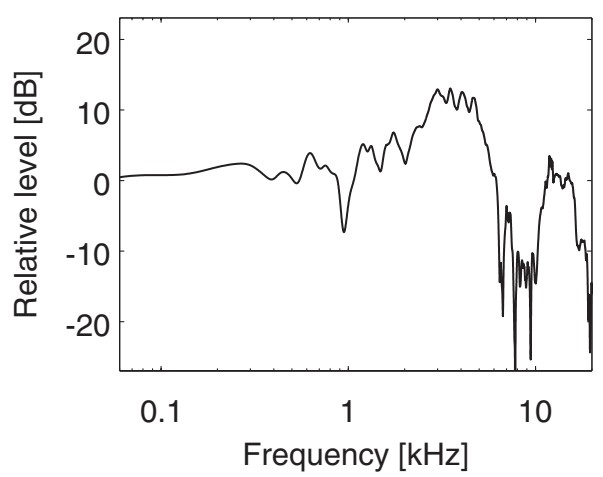

(a) original HRTF

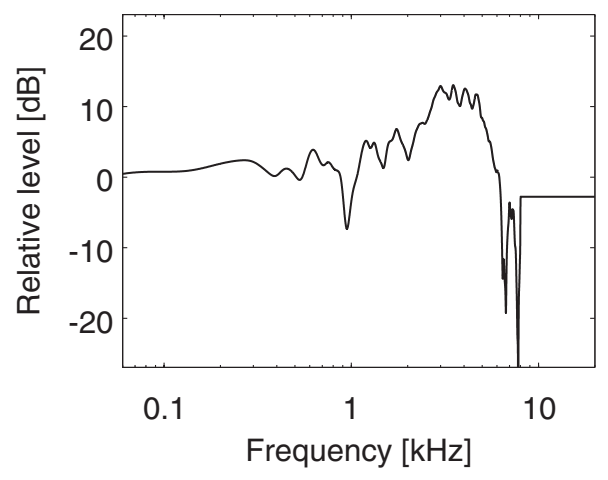

(b) flattened HRTF (boundary frequency: $8 \mathrm{kHz}$ )

Fig. 1 Example of the method for flattening contralateral HRTFs (source direction was diagonally forward right, left ear, boundary frequency: $8 \mathrm{kHz}$ ). The level in the flattened region is $-2.8 \mathrm{~dB}$, which is the same as the average level of the original HRTF in the frequency region corresponding to the flattened one.

one. Consequently, the overall ILD was retained in all directions.

\section{Evaluation experiment}

\subsection{Outline}

A localization test was conducted to evaluate the influence of flattening the contralateral HRTFs. Subjects seated in an anechoic chamber listened to a stimulus with headphones (HD-650; Sennheiser Electronic GmbH and Co. $\mathrm{KG})$. Each stimulus was a sound signal convolved with either the subject's own HRTFs or those with the contralateral HRTF flattened above the boundary frequency. The subjects were asked to judge the perceived direction in the horizontal plane by clicking a mouse on the corresponding position on a circle displayed on a computer screen, where the center of the circle corresponded to the head of the subject.

\subsection{Conditions}

Four male subjects with normal hearing acuity participated in the experiment. A set of HRTFs was measured for each subject in the anechoic chamber. In this experiment, the sound source azimuth of $0^{\circ}$ was defined as the front, and the others were defined in a clockwise rotation. Consequently, the contralateral side for the right ear corresponded to source directions greater than $180^{\circ}$, while that for the left ear corresponded to those below $180^{\circ}$. The interval of source

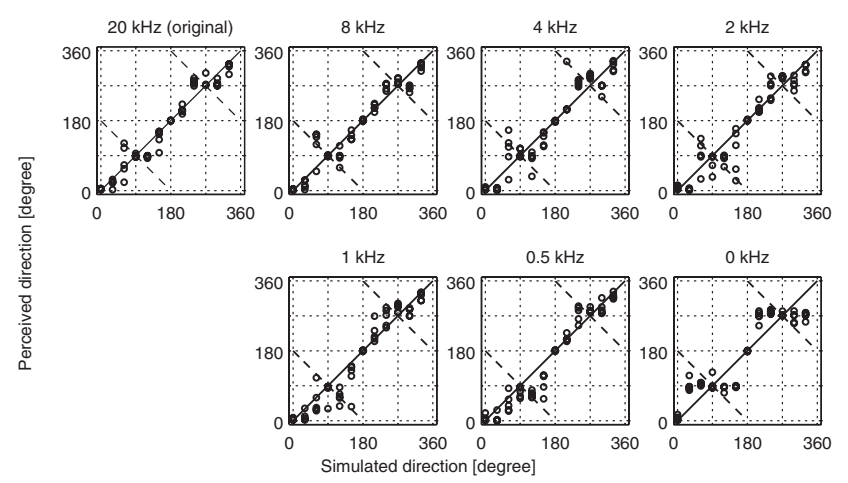

(a) subject 1

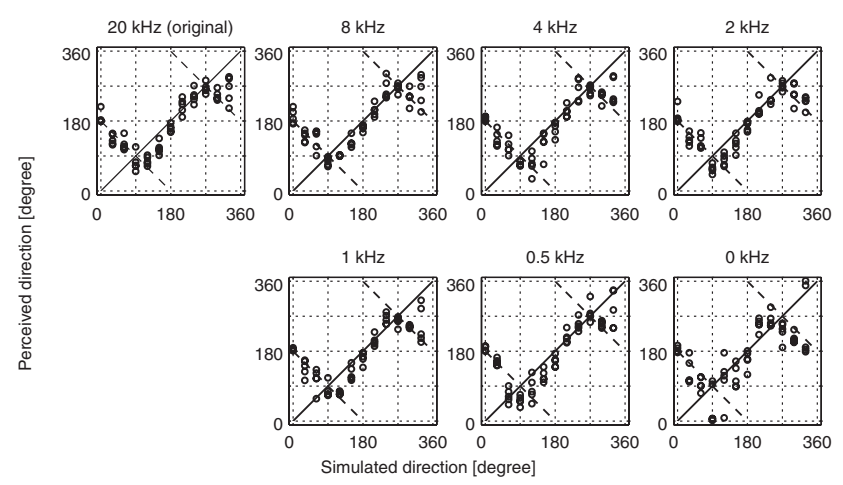

(b) subject 4

Fig. 2 Examples from two subjects of scatterplots of simulated directions versus subjects' perceived directions (Subs. 1 and 4).

directions was $30^{\circ}$ in the horizontal plane. Therefore, the number of source directions was 12. Each source signal consisted of pink noise with a duration of $1 \mathrm{~s}$ convolved with an HRTF; the ipsilateral stimulus was convolved with a measured HRTF while that of the contralateral side was convolved with a measured or flattened HRTF. The boundary frequencies were 20 (original), 8, 4, 2, 1, 0.5, and 0 (flattened for all frequency ranges) $\mathrm{kHz}$. Flattening was not applied to the HRTFs for two source directions, $0^{\circ}$ and $180^{\circ}$, because the contralateral side could not be defined. Each stimulus was evaluated five times in random order.

\section{Results and discussion}

\subsection{Results}

For original HRTFs, the localizations observed with subjects 1 and 2 were comparatively accurate, while those of subjects 3 and 4 were inaccurate for frontal directions. The results of two subjects for each condition are presented in Figs. 2(a) and 2(b) (subjects 1 and 4). The abscissa is the simulated direction and the ordinate is the direction perceived by the subject. The boundary frequencies are shown at the top of each panel.

In the " $20 \mathrm{kHz}$ " condition, the responses of subject 1 lay near the diagonal line, while those of subject 4 indicated front-back confusion, i.e., the subject tended to perceive a sound image from the frontal direction as being presented from the rear. Therefore, localization tendencies were differ- 


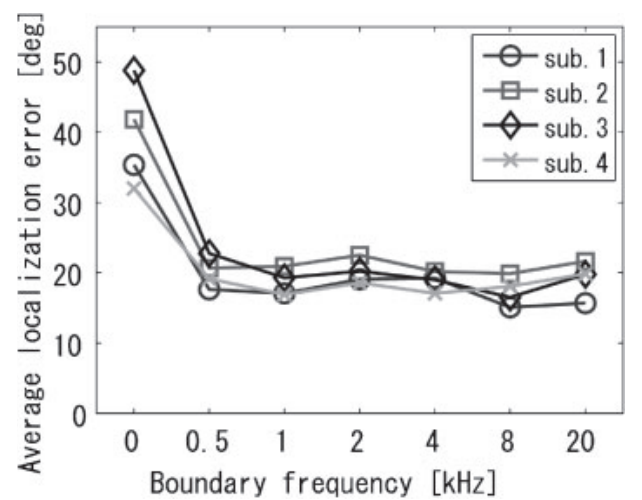

Fig. 3 Average localization error for various boundary frequency conditions.

ent among subjects. A similar pattern was observed with results associated with flattening of the HRTFs. These showed that the lower the boundary frequency, the more scattered the subjects' answers were, i.e., their localization performance degraded. In the " $0 \mathrm{kHz}$ " condition, however, the perceived directions were concentrated to either side $\left(90^{\circ}\right.$ or $\left.270^{\circ}\right)$ for some subjects. To evaluate the results of localization for the flattened HRTFs, localization errors and front-back confusion rates were analyzed.

4.2. Analysis of variance for localization error and frontback confusion

In this paper, localization error was defined as the absolute value of the difference between a source direction and a perceived direction; if front-back confusion occurred, the perceived direction was reversed relative to the direction that was symmetrical about the interaural axis [8]. Errors were calculated for all source directions. Front-back confusion rates were calculated for all source directions except $90^{\circ}$ and $270^{\circ}$. Figure 3 shows localization errors for each subject averaged over source directions. The abscissa is the condition of the flattened HRTF while the ordinate is the average localization error. The effects of the flattening, source directions, and subjects were analyzed using analysis of variance (ANOVA). The main effect of the flattened HRTFs was statistically significant $(F(6,207)=6.08, p<0.01)$. The main effect of the source directions was also significant $(F(9,207)=6.08$, $p<0.01)$. However, the main effect of the subjects was not significant. Because the main effect of the flattening was significant, the honestly significant difference (HSD) test was applied for multiple comparison. The main purpose of this study was to investigate the influence of flattening contralateral HRTFs. Therefore, we focused on the difference between errors in the " $20 \mathrm{kHz}$ " condition (i.e., the non-flattening condition) and each of the other conditions. Table 1 shows the conditions that were significantly different from the " $20 \mathrm{kHz}$ " condition, indicated by the asterisk $(*)$. Flattening contralateral HRTFs had a significant influence only in the " $0 \mathrm{kHz}$ " condition.

As described in Sect. 4.1, two subjects showed front-back confusion regarding the sound image from the frontal direction, even in the " $20 \mathrm{kHz}$ " condition. However, subjects experienced little front-back confusion regarding the sound image from the rear direction in the " $20 \mathrm{kHz}$ " condition.
Table 1 Results of the HSD test for the " $20 \mathrm{kHz}$ " condition. The asterisk indicates a significant difference was found for localization error.

\begin{tabular}{cllllll}
\hline Boundary frequency $[\mathrm{kHz}]$ & 0 & 0.5 & 1 & 2 & 4 & 8 \\
\hline Significance & $*$ & & & & & \\
\hline
\end{tabular}

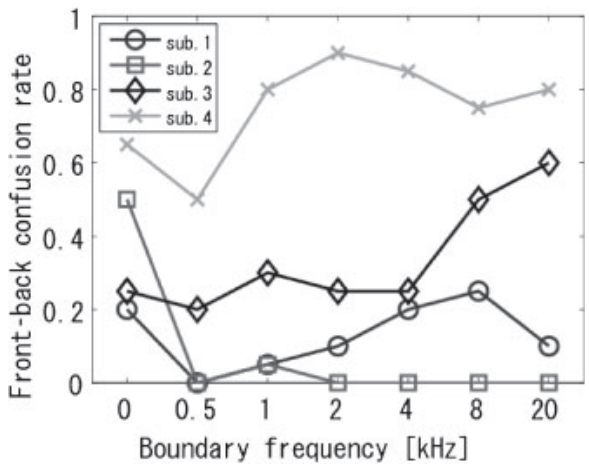

(a) Front-to-back confusion

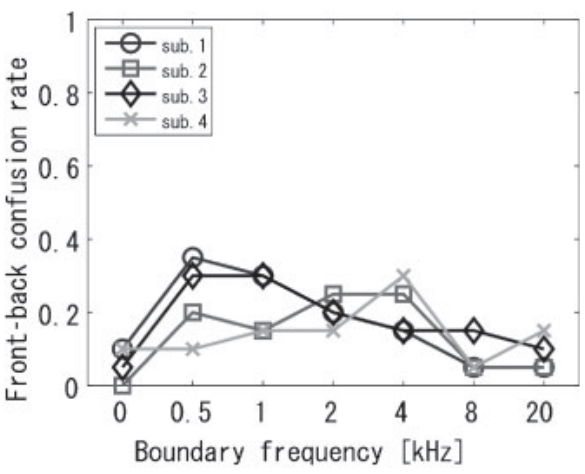

(b) Back-to-front confusion

Fig. 4 Front-back confusion rates for various boundary frequency conditions. Confusion rates are divided into two types: (a) "front-to-back confusion," which is defined as the error rate when subjects localized a frontally located source as coming from back, and (b) "back-to-front confusion," which is defined as the opposite case.

Figure 4 shows the front-back confusion rates for each subject for all source directions. The abscissa is the condition of the flattened HRTF and the ordinate is the front-back confusion rate. Figure 4(a) presents "front-to-back confusion," which is the rates for frontal source directions (from $0^{\circ}$ to $90^{\circ}$ and from $270^{\circ}$ to $360^{\circ}$ ). Figure 4 (b) shows the rate of "back-to-front confusion," i.e., when the source directions were from the back (from $90^{\circ}$ to $270^{\circ}$ ). The results of ANOVA on the effects of the flattened HRTFs, source directions (front or back), and subjects showed that the interaction between flattened HRTFs and source directions was significant $(F(6,18)=3.24$, $p<0.05)$. Because the simple main effect was significant only for the flattening $(F(6,18)=4.70, p<0.01)$ in rearward source directions, the HSD test was applied to flattened HRTFs of back directions for multiple comparison. Those conditions that showed significantly higher front-back con- 
Table 2 Results of the HSD test for the " $20 \mathrm{kHz}$ " condition. The asterisk indicates a significant difference was found for back-to-front confusion.

\begin{tabular}{ccccccc}
\hline Boundary frequency $[\mathrm{kHz}]$ & 0 & 0.5 & 1 & 2 & 4 & 8 \\
\hline Significance & & $*$ & $*$ & & $*$ & \\
\hline
\end{tabular}

fusion rates than the " $20 \mathrm{kHz}$ " condition are indicated by asterisks in Table 2.

\subsection{Discussion}

Figure 3 presents the influence of flattening on horizontal localization if front-back confusion is ignored. It is considered that the HRTF spectral forms on the contralateral side in frequency regions higher than $0.5 \mathrm{kHz}$ do not significantly influence localization error. The results imply that subjects were able to use the ILDs and ITDs as cues for sound localization because they were retained in the flattened HRTFs. Localization performance was greatly degraded in the " $0 \mathrm{kHz}$ " condition, i.e., flattening of the contralateral HRTFs was applied to the overall frequency range. Although it remains unclear what caused the degradation in this experiment, lateralization might occur because subjects were unable to use the spectral cues conveyed in the HRTFs on the contralateral side, and it therefore might be difficult for them to discriminate azimuth differences perceived internally. However, the importance of spectral cues included in the frequency region lower than $0.5 \mathrm{kHz}$ is also unclear. Additional experiments in which the boundary frequencies are set between $0 \mathrm{kHz}$ and $0.5 \mathrm{kHz}$ are needed to further clarify this issue.

The influence of flattening on localization is unclear in the results on front-to-back confusion shown in Fig. 4(a). However, Table 2 implies that back-to-front confusion is not influenced when the flattening is applied to frequency range over $8 \mathrm{kHz}$. Langendijk and Bronkhorst [8], who used a flattening method similar to ours, reported that front-back confusions significantly increased when spectral cues in the $5.7 \mathrm{kHz}$ to $11.3 \mathrm{kHz}$ or $8 \mathrm{kHz}$ to $16 \mathrm{kHz}$ frequency regions were removed. Other studies with approaches different from ours demonstrated the existence of spectral cues in frequency regions higher than $8 \mathrm{kHz}$. For instance, Hebrank and Wright [3] found the cue for localization of back elevation may exist in the $10 \mathrm{kHz}$ to $12 \mathrm{kHz}$ band. Iida et al. [7] argued that one of the two notches existing approximately between $8 \mathrm{kHz}$ and $10 \mathrm{kHz}$ was important for elevation discernment. The results in this paper showed that flattening in the frequency region higher than $8 \mathrm{kHz}$ was unlikely to influence back-to-front confusion. The fact that the flattening was applied only to contralateral HRTFs suggests that the auditory system can discriminate front-back source directions to some extent when cues included in ipsilateral HRTFs are available. This does not mean that contralateral HRTFs are unnecessary for localization, however, because back-to-front confusion rates significantly increased when the boundary frequency was 0.5 , 1 , or $4 \mathrm{kHz}$. The rate of back-to-front confusion was low when the boundary frequency was $0 \mathrm{kHz}$ simply because subjects' localizations were lateral directions for which the rates were unable to be calculated, while the localization errors increased in this case.

\section{Conclusions}

In this paper, spectral cues included in contralateral HRTFs were removed by flattening frequency characteristics in high-frequency regions to investigate the relationships between cues and frequency characteristics. The results of localization tests yielded the following conclusions.

1) Ignoring front-back confusion, the spectral form of contralateral HRTFs in frequency ranges higher than $0.5 \mathrm{kHz}$ did not influence horizontal localization.

2) With regard to back-to-front confusion, removing cues from only contralateral HRTFs in frequency ranges higher than $8 \mathrm{kHz}$ had no effect.

The results of our experiment suggest that the auditory system is unlikely to utilize all spectral information from contralateral HRTFs when all spectral information is available from ipsilateral HRTFs. Therefore, it is necessary to take account of that fact in studies on spectral cues in human localization. It might be revealed that the auditory system requires much less spectral information than that included in HRTFs. Furthermore, an efficient representation of HRTFs, such as a data reduction method, might be realized in the future.

\section{Acknowledgement}

This work was partially supported by a Grant-in-Aid for Young Scientists (B) (21700140).

\section{References}

[1] J. Blauert, Spatial Hearing (The MIT Press, Cambridge, Mass., 1983).

[2] J. Blauert, "Sound localization in the median plane," Acustica, 22, 205-213 (1969).

[3] J. Hebrank and D. Wright, "Spectral cues used in the localization of sound sources on the median plane," J. Acoust. Soc. Am., 56, 1829-1834 (1974).

[4] F. L. Wightman and D. J. Kistler, "Headphone simulation of free-field listening. II: Psychophysical validation," J. Acoust. Soc. Am., 85, 868-878 (1989).

[5] F. Asano, Y. Suzuki and T. Sone, "Role of spectral cues in median plane localization," J. Acoust. Soc. Am., 88, 159-168 (1990).

[6] D. J. Kistler and F. L. Wightman, "A model of head-related transfer functions based on principal components analysis and minimum-phase reconstruction," J. Acoust. Soc. Am., 91, 16371647 (1992).

[7] K. Iida, M. Itoh, A. Itagaki and M. Morimoto, "Median plane localization using parametric model of the head-related transfer function based on spectral cues," Appl. Acoust., 68, 835-850 (2007).

[8] E. H. A. Langendijk and A. W. Bronkhorst, "Contribution of spectral cues to human sound localization," J. Acoust. Soc. Am., 112, 1583-1596 (2002).

[9] B. C. J. Moore, "Frequency difference limens for short-duration tones," J. Acoust. Soc. Am., 54, 610-619 (1973). 